\title{
Generalized tonic-clonic seizure after a taser shot to the head
}

\author{
Esther T. Bui MD, Myra Sourkes MD, Richard Wennberg MD
}

\section{ABSTRACT}

During a police chase on foot, a previously well police officer was hit mistakenly by a taser shot meant for the suspect. The taser gun had been fired once, sending 2 barbed darts into his upper back and occiput. Within seconds, the officer collapsed and experienced a generalized tonicclonic seizure with loss of consciousness and postictal confusion. Subsequent magnetic resonance imaging scans of the head and electroencephalograms were normal. The patient has experienced no recurrence of seizure over more than a year of follow-up. This report shows that a taser shot to the head may result in a brain-specific complication such as generalized tonic-clonic seizure. It also suggests that seizure should be considered an adverse event related to taser use.

Une version française de ce résumé est disponible à l'adresse www.cmaj.ca/cgi/content/full/180/6/625/DC1

CMAJ 2009;180(6):625-6

$\mathrm{T}$ he taser stun gun, manufactured by Taser International in Scottsdale, Arizona, is a weapon used increasingly among law enforcement personnel to temporarily incapacitate detainees. Questions have arisen in both the scientific literature and the lay press about the device's safety. ${ }^{1-3}$ In this article, we report the occurrence of a generalized tonic-clonic seizure in a person who received a taser shot to the head.

\section{Case report}

The patient was a previously well police officer in his $30 \mathrm{~s}$ who took part in a police chase involving a suspected robber. $\mathrm{He}$ and a colleague cornered the suspect, who initially appeared to surrender but then attempted an escape. The officer had begun to chase the suspect on foot when he experienced a sudden, severe pain in the back of his head. He later described the moment as feeling like he had been "hit by a bat." He recalled letting out a brief gasp before losing consciousness. He had no recollection of falling to the ground on top of the suspect. Police records indicate that the officer's colleague had fired a taser shot meant for the suspect but that the 2 copper darts had instead struck the officer in the occiput and upper back. The officer had been wearing an armoured vest. Imme- diately after being shot, he was found by his colleague to be unresponsive and foaming at the mouth. His eyes were rolled upward and he had generalized tonic-clonic movements with apnea lasting for about 1 minute. He did not have urinary incontinence. Postictally, he was initially confused and combative. Emergency medical services personnel were able to restrain him. They recorded a Glasgow Coma Score of 9 within 5 minutes after arrival; 5 minutes later, his score was 13 .

The patient's next memory was of being in the emergency department. During this period, he felt as if he were in "a bad dream." As he gradually regained orientation over the next few hours, he became aware of thoracic tightness that was aggravated by deep breaths, and a severe headache. He was monitored overnight, then discharged in stable condition.

The patient had no history of febrile or unprovoked seizures, head injuries, headaches, meningitis or encephalitis. He had no family history of seizures or of other neurologic or psychiatric conditions. His developmental history was normal. He was not taking any medications.

The results of a general physical and neurologic examination were normal. Results of routine blood tests were unremarkable except for an elevated leukocyte count of 12.9 (normal 3.6-11.0) $\times 10^{\circ} / \mathrm{L} 30$ minutes after the event (decreasing to $11.2 \times 10^{\circ} / \mathrm{L} 5$ hours later) and an elevated serum creatine kinase level of 580 (normal <232) U/L.

The patient returned to full-time work 5 days after the incident. He experienced persistent headaches, dizziness, back pain and chest tightness. Magnetic resonance imaging scans of the head (1.5 and 3 Tesla) as well as routine and 24-hour ambulatory electroencephalography were performed 1, 2 and 12 months after the seizure. All findings were normal.

A diagnosis of mild traumatic brain injury (concussion), in addition to provoked seizure, was considered after a neurologic consultation during assessment of the patient at a rehabilitation centre 6 months after injury. A psychiatric consultation 7 months after injury suggested an Axis I diagnosis of adjustment disorder with depressed and anxious mood. Formal neuropsychological testing performed 9 months after injury showed no definite evidence of cognitive impairment in any domain.

From the Division of Neurology, Krembil Neuroscience Centre, University Health Network, Toronto Western Hospital, University of Toronto, Toronto, Ont. 
The patient has not had further seizures since the injury more than 1 year ago. His symptoms of anxiety, difficulties concentrating, irritability, nonspecific dizziness and persistent headaches have not completely resolved. Treatment trials have included amitriptyline $50 \mathrm{mg}$ nightly, topiramate $25 \mathrm{mg}$ nightly, escitalopram $10 \mathrm{mg}$ nightly, almotriptan $12.5 \mathrm{mg}$ as needed and ibuprofen 200-400 mg as needed.

\section{Comments}

A taser stun gun is a device designed to temporarily immobilize a human target by delivering a direct-current type of shock through 2 barbed copper darts. The shock causes involuntary muscle contraction. Neuromuscular transmission is thought to be affected primarily at the level of the peripheral motor nerve, although studies have shown that stimulation of the spinal cord may occur with dart penetration as far away as the anterior torso. ${ }^{4.5}$ The muscle contraction induced by tasers is typically tonic, with retained consciousness, no clonic movements and no postictal confusion. ${ }^{4}$ The manufacturer's website estimates that a single shot lasts about 5 seconds, delivers 19 pulses per second with a typical charge of 100 microcoulombs per pulse, generates an average net current of 2 milliamperes and has an estimated peak voltage of 1300 volts. $^{6}$

The data are sparse on how this device may affect the central nervous system. A case has been reported involving intracranial penetration by a taser dart with loss of consciousness for 5 minutes. ${ }^{7}$ The person who had been struck recovered shortly afterward with a mild headache. No details were reported on whether a seizure occurred, although only 1 of the 2 darts struck the patient. ${ }^{7}$ Another case report describes cranial penetration by a taser dart (with the second dart found in a hair braid) with transient decreased consciousness; no further details were given. ${ }^{5}$ Other reports of secondary loss of consciousness related to taser shots have involved only cases of severe traumatic head injuries that resulted from falls during neuromuscular incapacitation. ${ }^{5}$

The description by witnesses of the event involving our patient is most compatible with a generalized tonic-clonic seizure. The loss of consciousness, clonic movements, foaming at the mouth and postictal confusion experienced by our patient differentiate the episode from the usual transient incapacitation induced by tasers. The taser current that passed to his brain from the dart in the occiput probably provoked the seizure directly, with a mechanism akin to that of seizures induced by electroconvulsive therapy. In electroconvulsive therapy, an initial charge of 38-60 millicoulombs is used, according to therapeutic protocol in the United States. ${ }^{8}$ It is plausible that a copper dart penetrating the scalp and discharging 95 pulses of 100 microcoulombs each could trigger a generalized convulsion.

Given previous case reports of taser-induced cardiac arrhythmias, one could speculate that an initial induced cardiac arrhythmia and a secondary hypoxic seizure, or convulsive syncope, occurred in our case. Convulsive syncope is believed to result from reticular disinhibition in the brainstem resulting from hypoxia-induced cortical dysfunction. ${ }^{9}$ However, this mechanism seems unlikely in this case, especially given that the points of impact of the taser darts were over the head and upper back and not the heart.

Even less likely is the possibility that the convulsion was induced by a concussion resulting from the direct physical impact of the darts or impact of the patient's head on the ground. Our patient's prolonged period of unresponsiveness and subsequent postictal confusion is not typical of a concussive convulsion, which is usually characterized by immediate onset and a rapid recovery that takes place over a few minutes. ${ }^{10}$ On the other hand, we believe that his persistent symptoms after injury may be attributable in part to postconcussion syndrome, presumably secondary to mild traumatic brain injury caused by either the impact of the taser dart or the subsequent fall to the ground during the provoked seizure.

Until now, most reports of taser-related adverse events have understandably concentrated on cardiac complications associated with shots to the chest. ${ }^{2}$ Our report shows that a taser shot to the head may result in brain-specific complications. It also suggests that seizure should be added to the list of taser-related adverse events.

This article has been peer reviewed.

Competing interests: None declared.

Contributors: All of the authors were involved in the preparation of this manuscript and approved the final version submitted for publication.

\section{REFERENCES}

1. Stanbrook MB, Hébert PC, Kale R, et al. Tasers in medicine: an irreverent call for proposals [editorial]. CMAJ 2008;178:1401-2.

2. Nanthakumar K, Massé S, Umapathy K, et al. Cardiac stimulation with high voltage discharge from stun guns. CMAJ 2008;178:1451-7.

3. Hume M, Dhillon S. Questions hang over taser death. Globe and Mail [Toronto] 2007 Oct. 26; Available: www.theglobeandmail.com/servlet/story/RTGAM .20071025.wtaser1026/BNStory/National/home (accessed 2009 Jan. 7).

4. Sun H, Webster JG. Estimating neuromuscular stimulation within the human torso with Taser stimulus. Phys Med Biol 2007;52:6401-11.

5. Mangus BE, Shen LY, Helmer SD, et al. Taser and taser associated injuries: a case series. Am Surg 2008;74:862-5.

6. Kroll MW. Cardiac safety. Scottsdale (AZ): Taser International; 2007. Available: www.taser.com/research/Science/Pages/CardiacSafety.aspx (accessed 2009 Jan. 9)

7. Rehman TU, Yonas H, Marinaro J. Intracranial penetration of a TASER dart. Am J Emerg Med 2007;733:e3-4.

8. Lisanby SH. Electroconvulsive therapy for depression. N Engl J Med 2007; 357:1939-45.

9. Stephenson JBP. Fits and faints. Oxford (UK): MacKeith Press, Blackwell; 1990. p. 41.

10. McCrory PR, Bladin PF, Berkovic SF. Retrospective study of concussive convulsions in elite Australian rules and rugby league footballers: phenomenology, aetiology, and outcome. BMJ 1997;314:171-4.

Correspondence to: Dr. Richard Wennberg, Toronto Western Hospital, Ste. 5W444, 399 Bathurst St., Toronto ON M5T 2S8; fax416 603-5768; r.wennberg@utoronto.ca 